\title{
Assessment of straylight and the modulation transfer function of intraocular lenses with centrally localized opacification associated with the intraocular injection of gas
}

Grzegorz Łabuz, ${ }^{1,} \mathrm{PhD}$, Timur M. Yildirim, ${ }^{1}$ MD, Thomas J.T.P van den Berg, ${ }^{2} \mathrm{PhD}$, Ramin Khoramnia, ${ }^{1}$ MD, FEBO, Gerd U. Auffarth, ${ }^{1}$ MD, PhD

${ }^{1}$ David J Apple Center for Vision Research, Department of Ophthalmology, University Hospital Heidelberg, INF 400, 69120 Heidelberg, Germany

${ }^{2}$ Netherlands Institute for Neuroscience, Royal Netherlands Academy of Arts and Sciences, Amsterdam, the Netherlands

Correspondence to the authors at: Grzegorz Łabuz, University Hospital Heidelberg, INF 400, 69120 Heidelberg, Germany. E-mail: g.labuz@hotmail.com

Financial Disclosures:

This research was supported by the Heika Project 2017 and the Klaus Tschira Foundation.

The Netherlands Academy of Arts and Sciences owns a patent on straylight measurement, with Van den Berg as the inventor, and licenses that to Oculus for the C-Quant instrument.

The authors have no proprietary or commercial interest in any materials discussed in this article. 


\begin{abstract}
PURPOSE: To assess the optical quality of intraocular lenses (IOLs) explanted, and submitted to the David J. Apple laboratory, because of lens opacification after the intraocular injection of gas.
\end{abstract}

SETTING: David J. Apple Laboratory, Heidelberg, Germany

DESIGN: An in vitro laboratory study.

METHODS: Four hydrophilic acrylic IOLs were studied, each with a centrally-localized round opacification pattern associated with the intraocular use of gas. Laboratory analysis included gross examination with a light microscope, followed by Alizarin and von Kossa staining. Optical quality was assessed by examining the modulation transfer function (MTF) and straylight. Results were compared with those of a control lens, and normative data for straylight of the crystalline lens. The following parameters were derived from image analysis: opacified surface fraction, light loss in the opacified surface, the area, and number of granules. The relationship between straylight increase and those parameters was studied.

RESULTS: Fine granules were identified on the IOL (sub)surface. The granules stained positive for Calcium. MTF levels of two IOLs dropped markedly. The other two showed relatively minor changes. Straylight was seriously increased in three IOLs up to (and above) a level of that of a cataractous lens. A proportional relationship was found between straylight and the morphological parameters from image analysis.

CONCLUSIONS: IOLs with centrally localized opacification have a strong potential for deteriorating optical performance. However, the optical quality may differ depending on the 
morphology of opacification. A serious straylight increase was found in most of these lenses suggesting that affected patients may suffer from glare related symptoms.

\section{Introduction}

Intraocular lens (IOL) opacification is a serious condition that may degrade patients' vision. ${ }^{1-18}$ Although opacification has often been associated with hydrophilic IOLs, 2, 5, 7, 9-15, 17, 18 this postoperative complication has also been reported in other lens materials. ${ }^{1,3,6,16}$ Studies on calcification of hydrophilic acrylic IOLs have pointed to several factors that may be related to the onset of opacification, such as lens packaging, ${ }^{4}$ ophthalmic viscosurgical device, ${ }^{8}$ surgical technique, ${ }^{19}$ patient related conditions, ${ }^{6}$ and most recently, intracameral injection of gas. ${ }^{2,5,7,9-15}$, ${ }^{17,}{ }^{18}$ IOL opacification due to the intraocular gas usage created a new type of centrally localized round calcification pattern on one lens (sub)surface and restricted to the pupillary or capsulorhexis area. $^{2,5,7,9-15,17,18}$ It has been reported following ophthalmic procedures involving the use of gas, such as posterior lamellar keratoplasty, e.g., Descemet-stripping (automated) endothelial keratoplasty (DS[A]EK) and Descemet membrane endothelial keratoplasty (DMEK), 5, 7, 10-15, 18, 20 and pars plana vitrectomy (PPV). ${ }^{2,}$, 17 However, the incidence rate is relatively low, ranging from $2.5 \%$ to $5 \% \cdot{ }^{13}, 15$ Opacification typically appears several months after those procedures, which may result in patients' complaints, and IOL exchange., 5, 9-12, 17, 18 Although numerous studies on histological examination have been performed, 2, 5, 7, 9-12, 17, 18 little can be found on the optical quality of the affected IOLs. ${ }^{7,11}$

The optical performance of opacified lenses has been studied by means of light scattering (straylight) measurements, ${ }^{21-23}$ and quality metrics derived from the point spread function, such as the modulation transfer function (MTF) and the Strehl ration (SR) ${ }^{24-27}$ Studies on straylight from different types of IOL opacification such as calcification, snow-flake degeneration, and 
glistenings, have shown that straylight is a very sensitive measure to detect the presence of IOL pathology. ${ }^{20,28,} 29$ Deterioration of the optical quality in those lenses in terms of the MTF, occurs only if opacification is severe. ${ }^{20,30}$ MTF levels of a calcified IOL after the DMEK/DS(A)EK procedure were presented in two studies, however, straylight was not included in those reports. ${ }^{12}$ 22

The aim of this laboratory study was to assess the effect of centrally localized IOL calcification, associated with the intraocular injection of gas, on the lens optical quality by measuring the MTF and straylight measurements. The relationship was established between the straylight parameter and morphological parameters, which were derived from the laboratory analysis and image processing.

\section{Methods}

Table 1 provides detailed information on four IOLs, which were explanted between Dec 2016 and Apr 2017 and sent to the David J Apple International Laboratory for Ocular Pathology (Heidelberg, Germany). Two patients had received a combined phaco-vitrectomy with the use of $\mathrm{C}_{2} \mathrm{~F}_{6}$ gas (case 1 and 2). One patient (case 3) underwent PPV with the instillation of $\mathrm{C}_{3} \mathrm{~F}_{8}$ a year and a half after initial crystalline lens extraction. In this case, a subsequent anterior chamber revision was carried out because of the gas in the anterior chamber. Case 4 involved the use of trypan blue and air during cataract surgery. A month after cataract surgery, a DMEK procedure was performed in this patient with subsequent re-bubbling in the next four days. The mean 
patient age on the day of IOL explanation was 53 (19 to 74) years. The mean time the IOL had been inside the eye was 2.9 (0.6 to 5.3) years.

Studied intraocular lenses

All studied lenses were CT Asphina 409M/MP (Carl Zeiss Meditec AG, Germany). The CT Asphina is a monofocal aberration-neutral IOL made of hydrophilic acrylic material with a hydrophobic surface. The nominal power of the studied lenses is presented in Table 1 . The optical performance of the lens explants was compared with a control CT Asphina 409MP lens with a nominal power of $21.0 \mathrm{D}$.

Straylight assessment

Light scattering was assessed using a commercial device (C-Quant; Oculus GmbH, Germany) adapted for in vitro straylight measurements of IOLs. ${ }^{21,22}$ In short, the straylight meter consists of a central test field surrounded by an annular straylight source with effective $7^{\circ}$ radius. ${ }^{31-33}$ It uses the two-alternative forced choice psychophysical compensation comparison method, which has been described extensively elsewhere. ${ }^{31,34}$ In a clinical setting the straylight test is performed monocularly with a subject looking at the test field through an ocular. In this laboratory setting an IOL was placed into a BSS-filled glass cuvette and mounted in front of the ocular. The cuvette was positioned in a holder right behind a plano-convex lens to project the C-Quant test field into the IOL in the same way as during in vivo straylight measurement. ${ }^{21}$ The straylight test 
is performed in the standard way by an observer. The observer's eye was, however, not exposed to the straylight source as that was blocked by a diaphragm. Hence, only straylight of the lens under test was measured. ${ }^{21,} 22$ In this manner, the straylight measured in vitro directly corresponds to the straylight (increase) experienced by the patient.

The straylight meter provides straylight values as the logarithm of the straylight parameter, i.e. $\log (\mathrm{s})$. In this study, however, $\log (\mathrm{s})$ values were converted to the straylight parameter (s) for the purpose of computational analysis. Straylight of the studied lenses was compared to a straylight level of a 20-year-old ( $\mathrm{s}=2.5 \mathrm{deg}^{2} / \mathrm{sr}$ ), 70-year-old ( $\left.\mathrm{s}=11.2 \mathrm{deg}^{2} / \mathrm{sr}\right)$ and cataractous (s=33.1 $\mathrm{deg}^{2} / \mathrm{sr}$ ) crystalline lens. ${ }^{35-37}$ Two measurements per lens were performed.

\section{Optical metrology}

In addition to the straylight measurements, the optical quality of each lens explant was studied with OptiSpheric IOL PRO 2 optical bench (Trioptics GmbH, Germany). The IOL PRO2 was designed in compliance with ISO $11979 .^{38}$ The device consists of a monochromatic light source (546 nm), a target (e.g., slit, pinhole), a model eye, a microscope objective lens, and a monochromatic camera. An aberration-free model cornea was used in this study, ${ }^{38}$ as the main goal was to investigate the extent to which the optical quality of the explanted lenses had changed.

The optical performance of the IOLs was assessed by means of the MTF, which is derived from the Fourier Transformation of the line spread function (LSF) projected by the lens under test. ${ }^{24,} 25$ The LSF is an aberrated image of a slit test target. The MTF was measured (two 
repetitions) with a horizontal and vertical slit, which corresponds to the sagittal and horizontal planes, respectively. MTF results of the two meridians were averaged and presented graphically for a 3- and 4.5-mm pupil. ${ }^{39}$ These values were also used to calculate a Strehl ratio (SR) in the frequency domain using the following formula ${ }^{24}$

$$
\text { Strehl ratio }=\frac{\iint_{-\infty}^{\infty} \operatorname{MTF}\left(f_{\text {sag }}, f_{\text {tan }}\right) d f_{\text {sag }} d f_{\text {tan }}}{\iint_{-\infty}^{\infty} D L M T F\left(f_{\text {sag }}, f_{\text {tan }}\right) d f_{\text {sag }} d f_{\text {tan }}}
$$

Where DLMTF stands for a diffraction-limited MTF, and $f$ is the spatial frequency. The cut-off frequency used to calculate the SR was $100 \mathrm{lp} / \mathrm{mm}^{38}$ (30 cyc/deg) as it corresponds to a logMAR visual acuity (VA) of 0 .

Laboratory analysis

Following the optical measurements, the IOLs were cut in halves and subjected to laboratory analysis. A light microscope (BX5, Olympus Optical Co. Ltd., Japan) equipped with a camera (C-7070, Olympus Optical Co. Ltd., Japan) was used for gross examination of the lens explants and image recording. The lenses were stained for Calcium by means of the Alizarin and von Kossa method using a protocol that has been described in detail in recent articles. ${ }^{7,11,30}$

Image analysis 
Images were processed with a purpose-written software (Image Processing Toolbox, Matlab, Mathworks). The area of opacification and the loss of light were derived from photographs taken with a monochromatic camera (VA-1MC-M120-A0-C, Vieworks, South Korea) of the IOL PRO 2 device. The calcification area was assessed based on the difference in intensity levels of individual pixels between the opacified and clear part. This value and the total lens area were used to calculate an opacified fraction of the lens:

$$
\text { fractionArea }=\frac{\text { Area of calcification }}{\text { Total lens area }}
$$

The pixel values of the affected and unaffected regions of the lenses were averaged to obtain the loss of light in the calcified area, and that is expressed as:

$$
\text { fractionLight loss }=1-\frac{\text { Mean pixel intensity of the calcified area }}{\text { Mean pixel intensity of the clear area }}
$$

As a simple model, one might assume that straylight is proportionally related to both these parameters. The product of these two parameters gives the fraction of light scattered. So, the relation between the straylight parameter and this product will be studied. In addition, images of the IOLs following the von Kossa staining were analyzed with a Matlab software to find the size and number of Calcium deposits. This program has previously been used for the assessment of glistening in IOLs. ${ }^{23}$ The product of these two parameters can be expected to be proportional to the fraction of light scattered and thus, this product can be compared with straylight

\section{Results}


A centrally localized round area of discoloration (opacification) was identified by gross examination in the four studied IOLs (Figure 1, top panels). Fine granular deposits were revealed with light microscopy, which stained positive for Calcium (Figure 1, middle and bottom panels). The calcium deposits were localized on the lens surface only in case 3 (Figure 1, middle panels), but subsurface inclusions were found in all studied lenses (Figure 1, bottom panels).

The area of opacification corresponded to a lens fraction of $0.08,0.39,0.45$ and 0.23 in case 1, 2, 3 and 4, respectively. The loss of light in the calcified region was 0.27 for case 1, 0.64 for case 2, 0.50 for case 3, and 0.45 for case 4 . The analysis of the Calcium granules revealed an average diameter of $4.1 \pm 4.8 \mu \mathrm{m}, 2.5 \pm 4.2 \mu \mathrm{m}, 2.1 \pm 4.1 \mu \mathrm{m}$, and $2.7 \pm 0.7 \mu \mathrm{m}$ in case $1,2,3$ and 4, respectively.

MTF and SR values for the 3- and 4.5-mm pupils are presented in Figure 2. The control IOL showed very good optical performance in terms of the MTF test, as the SR at the two apertures was close to 1 . In comparison to the control, two IOLs (case 2 and 3) showed a significant drop in the MTF levels, with a lower SR than that of the control lens by $48 \%$ and $51 \%$ at $3 \mathrm{~mm}$. For the $4.5-\mathrm{mm}$ pupil it was 34\% and 49\% for case 2 and 3, respectively. Case 1 and 4 showed a $4 \%$ decrease in the SR at the 3-mm aperture with respect to the control. For the larger aperture, the SR was worse than that of the control by $10 \%$ for case 1, and $21 \%$ for case 4 .

The results of the straylight assessment are presented in Figure 3. Only the control demonstrated a straylight level that was below that of a young crystalline lens. Although straylight of case 1 was higher than that of the clear IOL, it was still within the normative limits of an (aged) natural lens. In the remaining three IOLs straylight was higher than that of a lens with cataract. A directly proportional relationship of the straylight parameter with the 
fractionArea $\mathrm{x}$ fractionLight loss values, and the mean Calcium granule surface $\mathrm{x}$ the total number of Calcium granules appear in Figure 4.

\section{Discussion}

The main finding of the present study is that IOL calcification following the intracameral injection of gas caused serious deterioration of the lens optical performance in terms of straylight (disability glare) particularly, and MTF metrics in some cases (Figure 2 and 3). This loss of optical quality strongly depends on density and size of Calcium deposits, which act as lightscattering particles yielding the loss of light for the direct light transmission, and the area of calcification (Figure 4). The straylight study shows that the light lost for direct imaging is not actually lost, but enters into the eye, causing a strong disturbing light veil over the retina. The straylight values are huge compared to the effects on the MTF or SR.

The MTF of opacified lenses has been studied before. In one case report on IOL opacification subsequent to the DMEK procedure, a large effect on optical quality metrics was found with MTF levels close to zero at frequencies above $50 \mathrm{lp} / \mathrm{mm}$ (15 cyc/deg). ${ }^{11}$ This was confirmed by a later study on a larger number of calcified IOLs. ${ }^{7}$ In two other studies, on a distinct opacification pattern, a steep decline of MTF values was demonstrated. ${ }^{20,30}$ In the current study, not all analyzed lenses demonstrated significant MTF effects. The SR of two IOLs (case 1 and 4) was lower than that of the control lens only by 4\% at the 3-mm pupil. Although the SR of these two lenses was lower at the 4.5-mm pupil, it remained at a high level of 0.86 (case 1) and 0.75 (case 2). The optical (MTF) performance of the two other lenses decreased markedly with respect to the control lens at the two apertures. These findings suggest that the optical quality of calcified IOLs is variably maintained depending on the extent of the calcification. 
We found that straylight was seriously increased in three of the four lenses, also in case 4 where a moderate effect on the MTF was shown (Figure 2 and 3). These three IOLs demonstrated higher straylight levels than that of an average cataract lens. Straylight of one lens was below that level (case 1) with $\mathrm{s}=4.9 \mathrm{deg}^{2} / \mathrm{sr}$, which corresponds closely to the scattering effect of a 50-year-old crystalline lens. ${ }^{33,35,40}$ With respect to the control IOL, a 3-, 78-, 54- and 25 -fold straylight increase was found in case 1, 2, 3, and 4, respectively. These high straylight values may results in patients' complaints of hazy or "foggy" vision, ${ }^{18}$ the loss of contrast, or difficulties in dynamic light conditions, particularly in the presence of a strong source of light. ${ }^{32,}$ 33, 40, 41 Although in Case 1 straylight increase was moderate, it might have caused notable disturbance because the patient was only 19-years-old, and at this age straylight is normally low. The found serious straylight increase in 3 cases suggests that IOL opacification has a greater potential for increasing straylight than deteriorating the MTF, as a 2-fold drop in MTF levels was found in the most affected IOL (case 2). This, however, could have been expected as the MTF and straylight correspond to separate domains of the functional PSF. ${ }^{32,42}$ The MTF metrics relate strongly to the central part (up to 10 minutes of arc) of the PSF, which accounts for lower-order and higher-order aberrations. ${ }^{32,}{ }^{42}$ By contrast, the straylight meter assesses the PSF at an effective 7-degree angle (5 to 10 degrees). This part of the PSF has a different functional importance. $^{32,33,40,42,43}$

To the best of our knowledge, no study on straylight from calcified IOLs after the intracameral injection of gas has been published in the peer-reviewed literature. Two studies reported light scattering from lens explants, however, the etiology of the calcification process of those lenses differ from that of the IOLs included in the current study. ${ }^{20,28}$ Werner at al. ${ }^{20}$ studied 13 hydrophilic acrylic IOLs with a diffuse calcification pattern within the lens optics, which 
extended to the haptics in some cases: one silicone lens with dense central opacification that was explanted from a patient with asteroid hyalosis, and four PMMA lenses had snow-flake degeneration. The average straylight parameter of those IOLs at 5- to 10-degree angle was 47.9 $\mathrm{deg}^{2} / \mathrm{sr}$ for the hydrophilic IOLs, $38.0 \mathrm{deg}^{2} / \mathrm{sr}$ for the silicon IOL, and $39.4 \mathrm{deg}^{2} / \mathrm{sr}$ for the PMMA IOLs. ${ }^{20}$ In another study, van der Meulen et al. ${ }^{28}$ investigated two hydrophilic acrylic lenses with uniform opacity of the lens optic and haptics. They found a straylight parameter of 63.1 and $794.3 \mathrm{deg}^{2} / \mathrm{sr}$ in those two cases. ${ }^{28}$ Although we studied a distinct opacification pattern, the mean straylight parameter $\left(68.1 \mathrm{deg}^{2} / \mathrm{sr}\right)$ of the current study corresponds approximately to the mean straylight levels found in those studies, with the exception of one extreme case reported by van der Meulen et al. ${ }^{28}$

The preceding paragraph summarizes straylight values in differing types of IOL opacification. Our study showed that scattering effects of IOLs with opacification associated with the intraocular injection of gas may also differ (Figure 3 and 4). This may be because of the differences in surgical procedures that were performed in these cases. We assessed the relationship between straylight and the (apparent) light loss with respect to the area of calcification (Figure 4). We calculated the calcified area in relation to the total lens surface. However, in order to more closely mimic an in vivo situation, one would use the natural pupil area as a reference, given that the peripheral part of the lens in vivo is covered by the iris. Unfortunately, this could not be achieved because neither pupil size measurements nor slit-lamp images prior to explantation were available to the authors. . The light observed in the microscopic image can be attenuated due to light scattering and/or absorption, depending on the properties of the relevant particles, ${ }^{44}$ in our case, Calcium granules. It was therefore interesting to investigate the relationship between the straylight parameter and the size and number of Calcium 
deposits/inclusions. Although the sample size was very small, a proportional relation seems to show up in Figure 4. However, one would expect a perfect proportional relation between these parameters. The reason for this could be the weighing of the light scattering process, which is always somewhat different from the weighing of the morphological parameters. For instance, we used images of $5-\mu \mathrm{m}$-thick IOL slices for the von Kossa test in our analysis. ${ }^{7,}$ 11, 30 However, Calcium granules are not evenly distributed within the IOL optic. Moreover, the size distribution and density of the Calcium granules showed a high degree of variability. Although this may appear as a limitation, increasing complexity of the model would not necessarily yield better accuracy, as scattering itself is a complex process. ${ }^{44}$

A recent clinical study on a large cohort of patients after the DMEK procedure showed a limited difference of $0.15 \log$ MAR between patient groups with and without lens opacification $(0.28 \pm 16$ vs. $0.13 \pm 8) .{ }^{15}$ IOL exchange was performed in one case in that report. ${ }^{15}$ Several studies on severe lens opacification and subsequent lens explantation have also indicated that the VA effects of opacification are often limited, with moderate $e^{5,10-12,17}$ or no ${ }^{17}$ VA improvement upon surgery. However, one case of regaining full VA was reported by MacLean et al. as well. ${ }^{10}$ One may expect a considerable improvement in visual function though in terms of disability glare, as the straylight effects of these lenses can be large. Clinical studies have demonstrated that straylight correlates poorly with VA, e.g. increased straylight was found in patients with good VA and vice versa. ${ }^{32,33,40,42,43}$ Therefore, a routine straylight assessment of patients with opacification may improve the decision-making process by increasing knowledge of potential benefits, as VA is not the sole indicator of visual function. ${ }^{32,33,40-43,45,46}$

The histological examination of the studied lenses revealed localized Calcium deposits/inclusions confined to one lens surface. Other authors have also reported a similar 
opacification pattern in the literature..$^{2,5,7,9-14,17,18}$ Dhital et al. ${ }^{2}$ investigated three cases of IOL opacification associated with the intracameral injection of gas. In one case, the size of opacities was $15 \mu \mathrm{m}$; in case 2 and 3 it was between 1 and $2 \mu \mathrm{m} .^{2}$ The mean size of the Calcium deposits we measured was less variable and ranged from 2.1 to $4.1 \mu \mathrm{m}$. Scattering particles of this size, being much larger than a wavelength, tend to scatter light in a forward direction (Mie theory). ${ }^{44}$ For the patient, forward scattering is more disturbing than backward scattering (as observed with a slit lamp) given that the forward scattered light falls onto the retina and may cause glare symptoms. ${ }^{32}$ Schrittenlocher et al. ${ }^{15}$ found a mean calcification area of $1.26 \pm 2.07 \mathrm{~mm}^{2}$ and 1.66 $\pm 2.48 \mathrm{~mm}^{2}$ following DMEK with miosis and mydriasis, respectively. However, only one (out of 14) calcified IOLs was replaced in that study group. In our study the values were $8.1 \pm 4.8 \mathrm{~mm}^{2}$ and all the lenses were exchanged. This suggests that IOLs with a larger area of opacification are more likely to affect the patient's vision and thus provoke subjective complaints that in turn lead the clinician to consider IOL exchange

Although only one IOL model was included in this study, IOL opacification following the intraoperative use of gas has been associated with various hydrophilic acrylic lenses. ${ }^{2,5,7,9-15,}$ 17, 18 Interestingly, one study reported on two hydrophobic lenses with post-DMEK opacification of a similar pattern. ${ }^{15}$ Therefore, the intracameral injection of gas appears as a risk factor for IOL calcification in various lens materials. However, the incidence of this postoperative complication seems to be low. ${ }^{13,15}$

In summary, we showed that centrally localized round IOL opacification, subsequent to the intraocular gas usage, can worsen the lens optical performance in terms of straylight and the MTF metrics. A proportional relation was shown between straylight and the morphological parameters explaining existing differences in optical quality between calcified lenses. The reason 
for the high variability found in the morphology of opacification between IOLs remains to be identified. The high straylight values reported may suggest increased sensitivity to glare of the affected patients resulting in a poor overall quality of vision independently of VA.

\section{Acknowledgements}

The authors would like to thank Frank G. Holz, MD, PhD (Universitäts Augenklinik, Bonn, Germany) and Silvia Bopp, MD, PhD (MVZ Universitätsallee Augenklinik, Bremen, Germany) for sending their IOL explants to the David J. Apple International Laboratory for Ocular Pathology.

\section{WHAT WAS KNOWN}

- A centrally localized calcification pattern on the (sub)surface of hydrophilic acrylic IOLs has been associated with the intracameral injection of gas. The optical (MTF) performance of the affected IOLs has been found to be affected with limited functional (acuity) effects, but straylight effects have not yet been established.

\section{WHAT THIS PAPER ADDS}

- The IOLs with localized opacification showed potential for degrading the optical performance. Degradation is limited in terms of the MTF and very strong in terms of straylight. 
- A proportional relationship between straylight and the morphological characteristics of opacities may explain variability in the optical quality of affected IOLs.

\section{References}

1. Apple DJ, Peng Q, Arthur SN, Werner L, Merritt JH, Vargas LG, Hoddinott DS, Escobar-Gomez M, Schmidbauer JM. Snowflake degeneration of polymethyl methacrylate posterior chamber intraocular lens optic material: a newly described clinical condition caused by unexpected late opacification of polymethyl methacrylate. Ophthalmology 2002;109:1666-75.

2. Dhital A, Spalton DJ, Goyal S, Werner L. Calcification in hydrophilic intraocular lenses associated with injection of intraocular gas. American journal of ophthalmology 2012;153:115460. e1.

3. Dogru M, Tetsumoto K, Tagami Y, Kato K, Nakamae K. Optical and atomic force microscopy of an explanted AcrySof intraocular lens with glistenings. J Cataract Refract Surg 2000;26:571-5.

4. Dorey MW, Brownstein S, Hill VE, Mathew B, Botton G, Kertes PJ, El-Defrawy S. Proposed pathogenesis for the delayed postoperative opacification of the hydroview hydrogel intraocular lens. American journal of ophthalmology 2003;135:591-8.

5. Fellman MA, Werner L, Liu ET, Stallings S, Floyd AM, Van Der Meulen IJ, LapidGortzak R, Nieuwendaal CP. Calcification of a hydrophilic acrylic intraocular lens after Descemet-stripping endothelial keratoplasty: case report and laboratory analyses. Journal of Cataract \& Refractive Surgery 2013;39:799-803.

6. Foot L, Werner L, Gills JP, Shoemaker DW, Phillips PS, Mamalis N, Olson RJ, Apple DJ. Surface calcification of silicone plate intraocular lenses in patients with asteroid hyalosis. Am J Ophthalmol 2004;137:979-87.

7. Giers BC, Tandogan T, Auffarth GU, Choi CY, Auerbach FN, Sel S, Mayer C, Khoramnia R. Hydrophilic intraocular lens opacification after posterior lamellar keratoplasty - a material analysis with special reference to optical quality assessment. BMC Ophthalmol 2017;17:150.

8. Jensen MK, Crandall AS, Mamalis N, Olson RJ. Crystallization on intraocular lens surfaces associated with the use of Healon GV. Arch Ophthalmol 1994;112:1037-42.

9. Lee SJ, Choi JH, Sun HJ, Choi KS, Jung GY. Surface calcification of hydrophilic acrylic intraocular lens related to inflammatory membrane formation after combined vitrectomy and cataract surgery. Journal of Cataract \& Refractive Surgery 2010;36:676-81. 
10. MacLean KD, Apel A, Wilson J, Werner L. Calcification of hydrophilic acrylic intraocular lenses associated with intracameral air injection following DMEK. Journal of Cataract \& Refractive Surgery 2015;41:1310-4.

11. Milojcic C, Latz C, Tandogan T, Auffarth G, Holz F, Choi C, Khoramnia R. Eintrübung einer hydrophilen Acryl-Intraokularlinse nach DMEK. Der Ophthalmologe 2016:1-6.

12. Mojzis P, Studeny P, Werner L, Piñero DP. Opacification of a hydrophilic acrylic intraocular lens with a hydrophobic surface after air injection in Descemet-stripping automated endothelial keratoplasty in a patient with Fuchs dystrophy. Journal of Cataract \& Refractive Surgery 2016;42:485-8.

13. Nieuwendaal CP, Van Der Meulen IJ, Patryn EK, Werner L, Mourits MP, Lapid-Gortzak R. Opacification of the intraocular lens after descemet stripping endothelial keratoplasty. Cornea 2015;34:1375-7.

14. Park JC, Habib NE, Moate RM. Intraocular lens opacification after corneal endothelial keratoplasty: electron microscopy and $\mathrm{x}$-ray element spectroscopy analysis. Journal of Cataract \& Refractive Surgery 2015;41:140-5.

15. Schrittenlocher S, Penier M, Schaub F, Bock F, Cursiefen C, Bachmann B. Intraocular Lens Calcifications After (Triple-) Descemet Membrane Endothelial Keratoplasty. American Journal of Ophthalmology 2017.

16. van der Mooren M, Steinert R, Tyson F, Langeslag MJ, Piers PA. Explanted multifocal intraocular lenses. J Cataract Refract Surg 2015;41:873-7.

17. Walker NJ, Saldanha MJ, Sharp JA, Porooshani H, McDonald BM, Ferguson DJ, Patel CK. Calcification of hydrophilic acrylic intraocular lenses in combined phacovitrectomy surgery. Journal of Cataract \& Refractive Surgery 2010;36:1427-31.

18. Werner L, Wilbanks G, Nieuwendaal CP, Dhital A, Waite A, Schmidinger G, Lee WB, Mamalis N. Localized opacification of hydrophilic acrylic intraocular lenses after procedures using intracameral injection of air or gas. Journal of Cataract \& Refractive Surgery 2015;41:199207.

19. Gartaganis SP, Kanellopoulou DG, Mela EK, Panteli VS, Koutsoukos PG. Opacification of hydrophilic acrylic intraocular lens attributable to calcification: investigation on mechanism. Am J Ophthalmol 2008;146:395-403.

20. Werner L, Stover JC, Schwiegerling J, Das KK. Effects of Intraocular Lens Opacification on Light Scatter, Stray Light, and Overall Optical Quality/PerformanceEffects of IOL Opacification on Stray Light. Investigative ophthalmology \& visual science 2016;57:3239-47.

21. Labuz G, Vargas-Martin F, van den Berg TJ, Lopez-Gil N. Method for in vitro assessment of straylight from intraocular lenses. Biomed Opt Express 2015;6:4457-64.

22. Labuz G, Papadatou E, Vargas-Martin F, Lopez-Gil N, Reus NJ, van den Berg T. Validation of a spectral light scattering method to differentiate large from small particles in intraocular lenses. Biomed Opt Express 2017;8:1889-94.

23. Labuz G, Reus NJ, van den Berg TJ. Straylight from glistenings in intraocular lenses: In vitro study. J Cataract Refract Surg 2017;43:102-8.

24. Thibos LN, Hong X, Bradley A. Metrics of optical quality of the eye. 2004.

25. Bass M, DeCusatis C, Enoch J, Lakshminarayanan V, Li G, Macdonald C, Mahajan V, Van Stryland E. Handbook of optics, Volume II: Design, fabrication and testing, sources and detectors, radiometry and photometry: McGraw-Hill, Inc.; 2009. 
26. Montes-Mico R, Madrid-Costa D, Ruiz-Alcocer J, Ferrer-Blasco T, Pons AM. In vitro optical quality differences between multifocal apodized diffractive intraocular lenses. J Cataract Refract Surg 2013;39:928-36.

27. Papadatou E, Del Aguila-Carrasco AJ, Esteve-Taboada JJ, Madrid-Costa D, MontesMico R. Assessing the in vitro optical quality of presbyopic solutions based on the axial modulation transfer function. J Cataract Refract Surg 2016;42:780-7.

28. Van der Meulen IJ, Porooshani H, van den Berg TJ. Light-scattering characteristics of explanted opacified Aquasense intraocular lenses. Br J Ophthalmol 2009;93:830-2.

29. Labuz G, Reus NJ, van den Berg TJ. Light scattering levels from intraocular lenses extracted from donor eyes. J Cataract Refract Surg (in press).

30. Tandogan T, Khoramnia R, Choi CY, Scheuerle A, Wenzel M, Hugger P, Auffarth GU. Optical and material analysis of opacified hydrophilic intraocular lenses after explantation: a laboratory study. BMC ophthalmology 2015;15:170.

31. Franssen L, Coppens JE, van den Berg TJ. Compensation comparison method for assessment of retinal straylight. Invest Ophthalmol Vis Sci 2006;47:768-76.

32. Van den Berg T, Franssen L, Coppens J. Ocular media clarity and straylight. Encyclopedia of the Eye 2010;3:173-83.

33. Van den Berg TJ, Franssen L, Kruijt B, Coppens JE. History of ocular straylight measurement: A review. Z Med Phys 2013;23:6-20.

34. Franssen L, Coppens JE, van den Berg TJ. Modulation depth threshold in the Compensation Comparison approach. J Vis 2007;7:8.

35. Vos JJ. Disability glare - a state of the art report. Commission International de l'Eclairage Journal 1984;3:39-53.

36. Vos JJ, Van Den Berg TJ. Report on disability glare. CIE collection 1999;135:1-9.

37. de Wit GC, Franssen L, Coppens JE, van den Berg TJ. Simulating the straylight effects of cataracts. J Cataract Refract Surg 2006;32:294-300.

38. ISO-11979-2. Part 2: Optical properties and test methods. In: Ophthalmic implants Intraocular lenses. Switzerland; 2014.

39. Koch DD, Samuelson SW, Haft EA, Merin LM. Pupillary and responsiveness: Implications for selection of a bifocal intraocular lens. Ophthalmology 1991;98:1030-5.

40. Van Den Berg TJ, Van Rijn LJ, Michael R, Heine C, Coeckelbergh T, Nischler C, Wilhelm H, Grabner G, Emesz M, Barraquer RI, Coppens JE, Franssen L. Straylight effects with aging and lens extraction. Am J Ophthalmol 2007;144:358-63.

41. Van den Berg TJ. On the relation between glare and straylight. Doc Ophthalmol 1991;78:177-81.

42. Berg TJ. The (lack of) relation between straylight and visual acuity. Two domains of the point-spread- function. Ophthalmic and Physiological Optics 2017;37:333 -41.

43. van der Meulen IJ, Gjertsen J, Kruijt B, Witmer JP, Rulo A, Schlingemann RO, van den Berg TJ. Straylight measurements as an indication for cataract surgery. J Cataract Refract Surg 2012;38:840-8.

44. van de Hulst HC. Light Scattering by Small Particles. New York: Dover Publications; 1981.

45. Elliott DB, Hurst MA, Weatherill J. Comparing clinical tests of visual function in cataract with the patient's perceived visual disability. Eye (Lond) 1990;4 ( Pt 5):712-7. 
46. Bradley A, Thomas T, Kalaher M, Hoerres M. Effects of spherical and astigmatic defocus on acuity and contrast sensitivity: a comparison of three clinical charts. Optom Vis Sci 1991;68:418-26.

\section{Figures}

Figure 1. Light microscopy images of the IOLs studied. Overview photographs (x1.25 magnification) of the lenses with a localized round opacification pattern are presented in the top panels. The middle panels show the IOLs stained with Alizarin; only in case 3 the Alizarin test was (unequivocally) positive indicating Calcium deposits on the anterior lens surface (x1.25 magnification). The results of the von Kossa staining method show (stained) Calcium inclusions (x40 magnification) within the lens optical body (the bottom panels).

Figure 2. The MTF (left panels) and the Strehl ration (right panels) values of the individual cases studied and compared with a control (clear) lens. The optical quality was measured at a 3-mm (top panels) and 4.5-mm pupil (bottom panels).

Figure 3. Straylight of the opacified IOLs measured at a 7-deg angle ( $\mathrm{sr}=$ steradians). The green and blue lines indicate a straylight effect of the crystalline lens at the age of 20 and 70 years, respectively. The red line represents an average straylight level of the lens with cataract.

Figure 4. Straylight parameter as a function of the mean Calcium granule surface times the total number of Calcium granules (black circles) and the opacified lens fraction (fractionArea) times the loss of light (fractionLight loss) on the opacified region (gray crosses). The dashed black and gray lines are proportionality lines. $\mathrm{Sr}=$ steradians. 

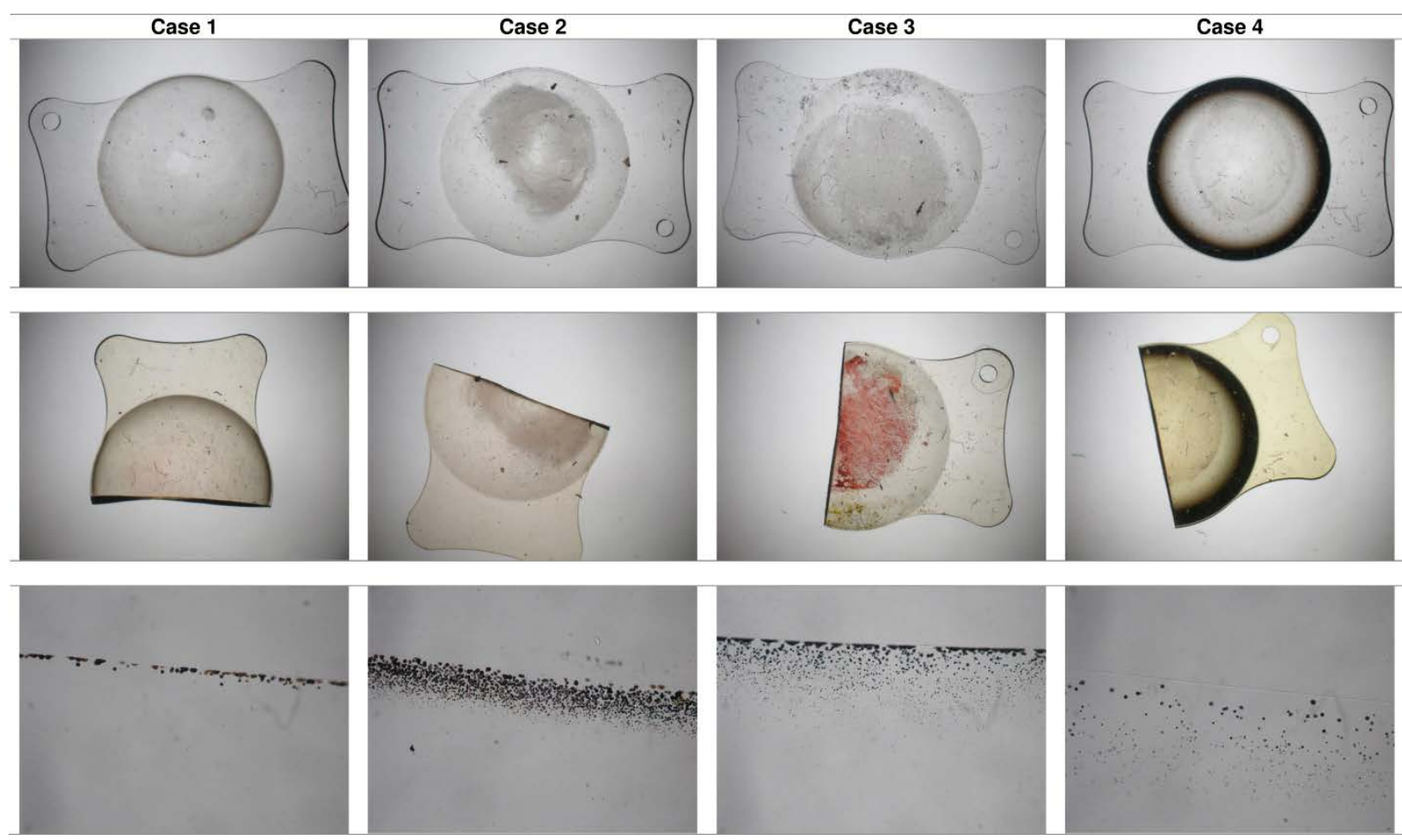
@ 3-mm aperture
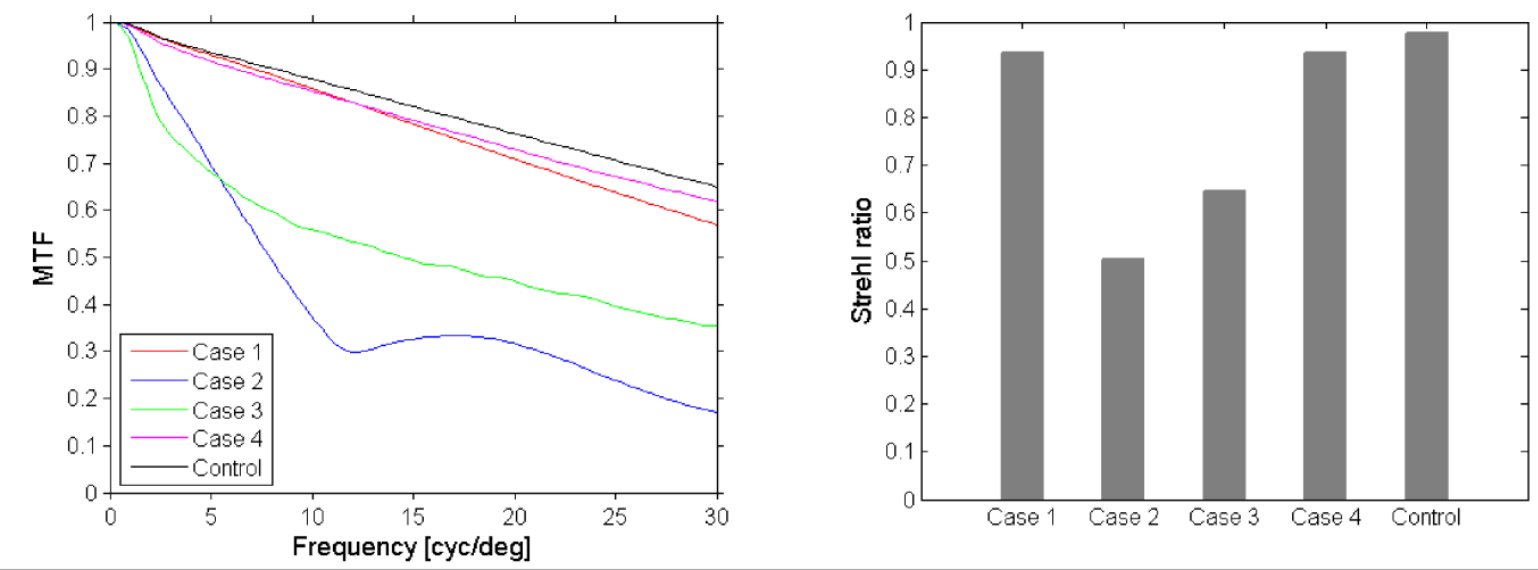

@ 4.5-mm aperture
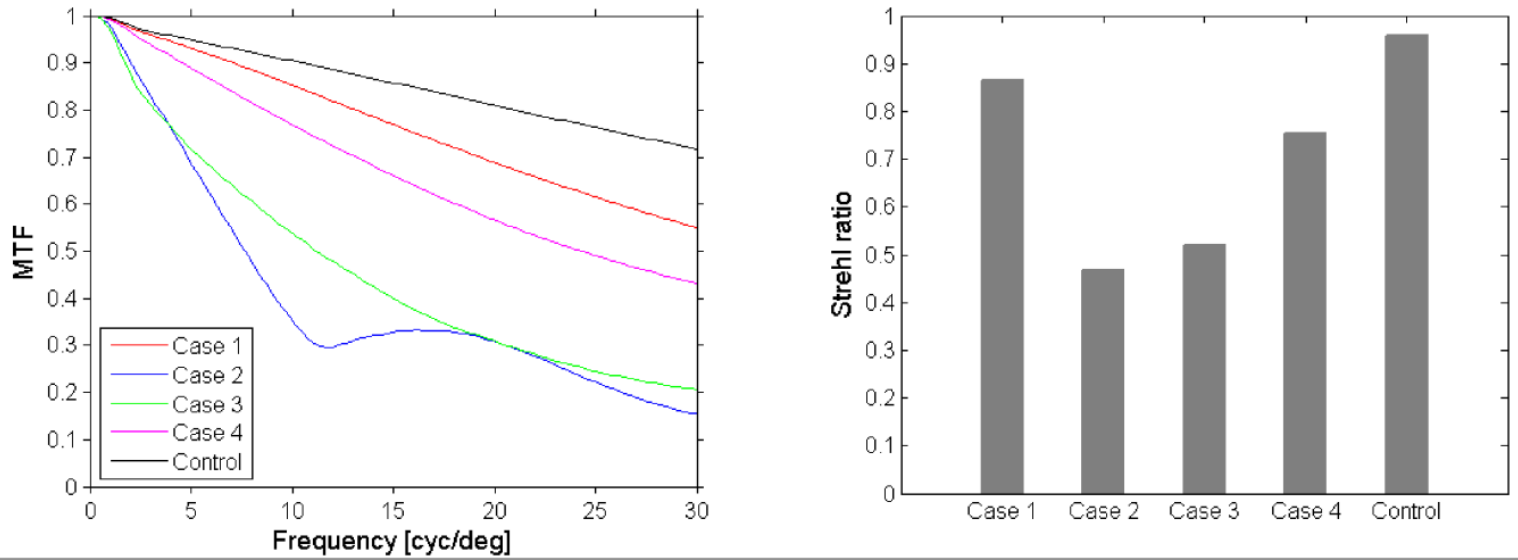


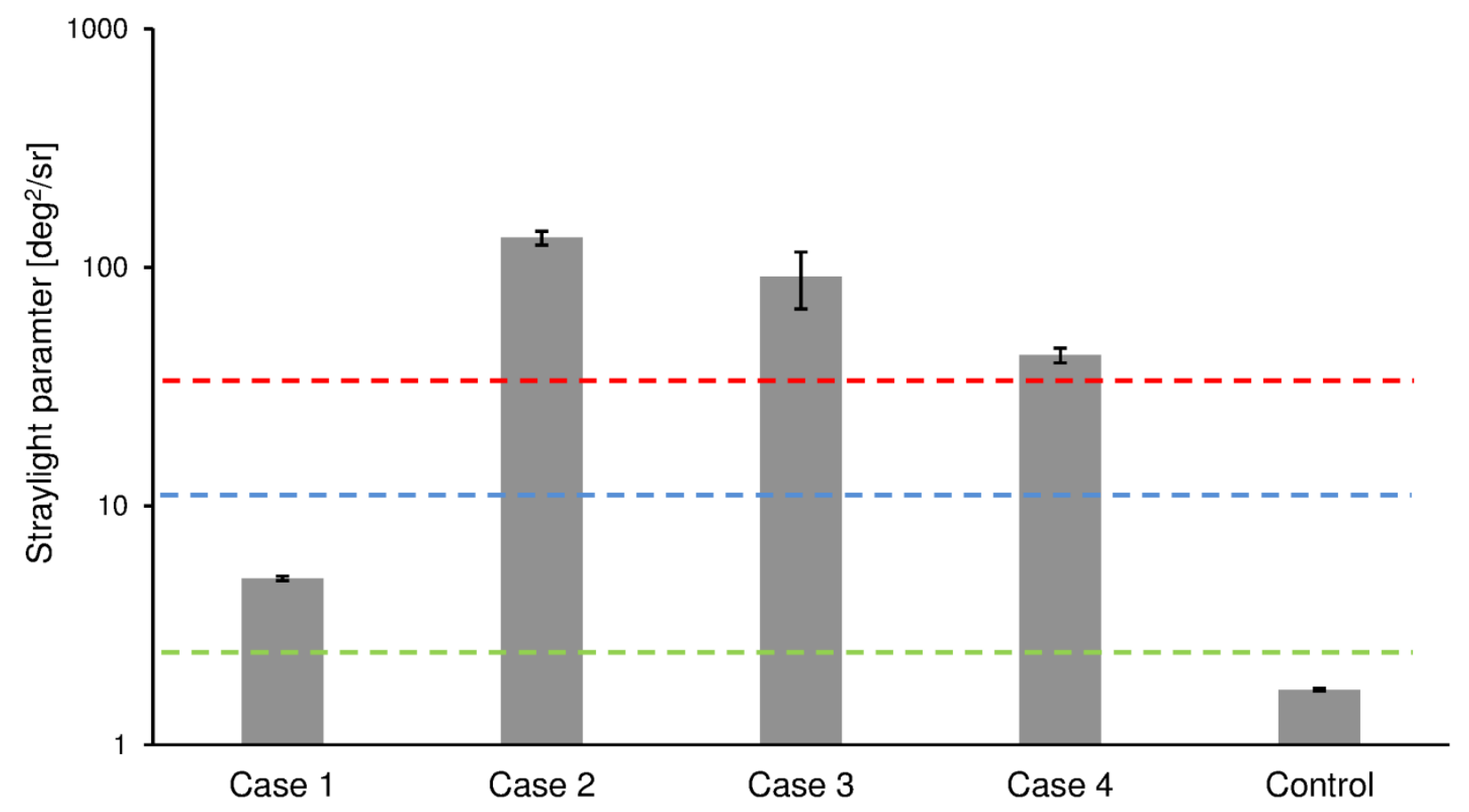




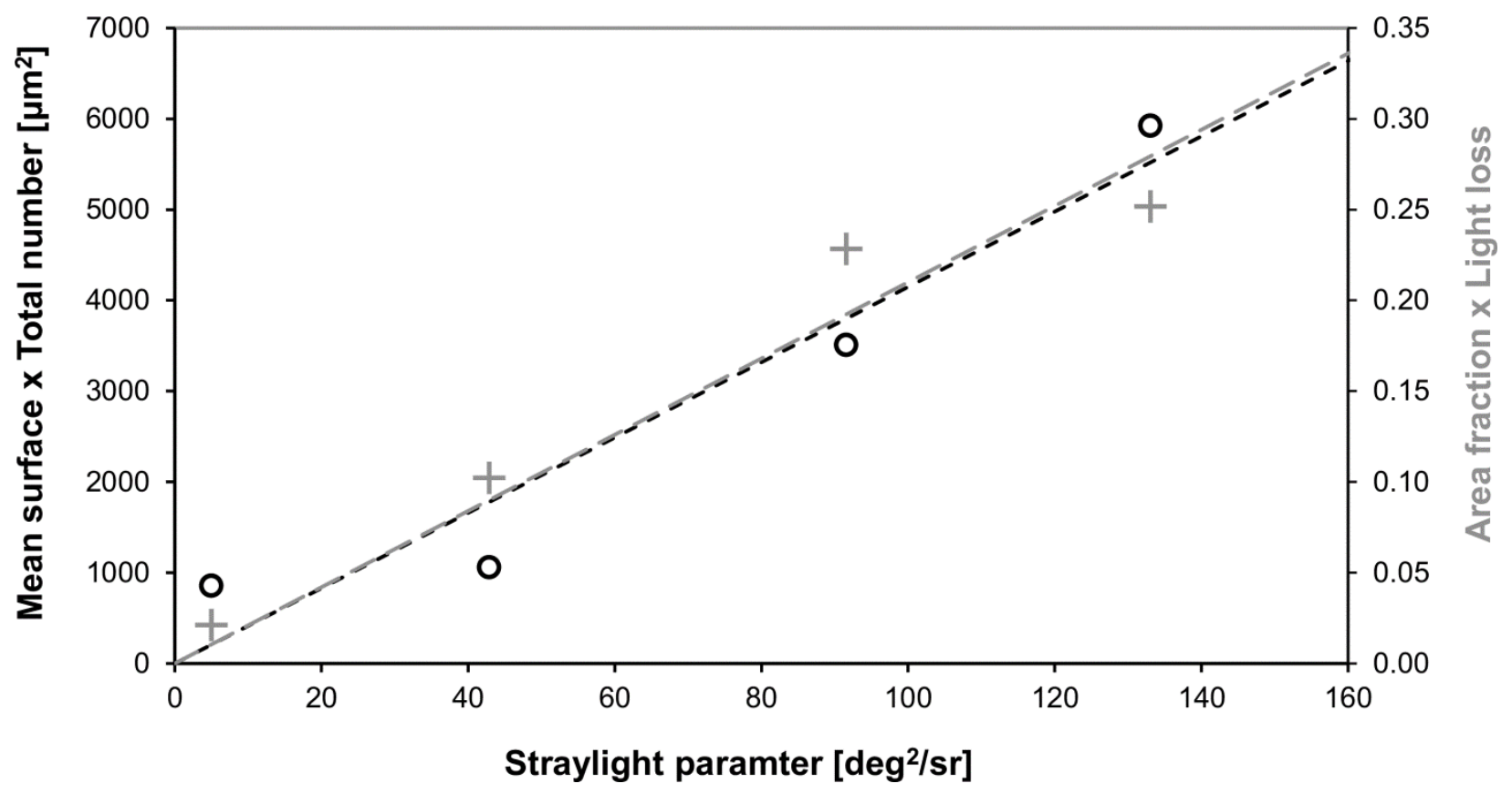




\begin{tabular}{|c|c|c|c|c|c|c|c|}
\hline Case & $\begin{array}{c}\text { IOL } \\
\text { Power [D] }\end{array}$ & $\begin{array}{l}\text { Implantation } \\
\text { date }\end{array}$ & $\begin{array}{l}\text { Explantation } \\
\text { date }\end{array}$ & $\begin{array}{l}\text { Time in } \\
\text { the eye } \\
{[\mathrm{Mo}]}\end{array}$ & $\begin{array}{c}\text { Patient age } \\
{[\mathrm{Y}]}\end{array}$ & $\begin{array}{c}\text { Associated } \\
\text { conditions }\end{array}$ & \\
\hline 1 & 20.0 & May 2016 & Dec 2016 & 7 & 19 & $\begin{array}{c}\text { Retinal } \\
\text { Detachment }\end{array}$ & Phaco-vitrectomy v \\
\hline 2 & 17.0 & Sep 2011 & Jan 2017 & 64 & 65 & $\begin{array}{c}\text { Retinal } \\
\text { Detachment }\end{array}$ & Phaco-vitr \\
\hline 3 & 16.5 & Nov 2013 & Mar 2017 & 40 & 57 & $\begin{array}{c}\text { Retinal } \\
\text { Detachment }\end{array}$ & $\begin{array}{r}\text { Phacoemulsification } \\
\text { 2015), gas in the at } \\
\text { YAG-l }\end{array}$ \\
\hline 4 & 25.0 & Sep 2014 & Apr 2017 & 31 & 74 & $\begin{array}{l}\text { Fuchs' } \\
\text { endothelial } \\
\text { dystrophy }\end{array}$ & $\begin{array}{l}\text { Phacoemulsifica } \\
\text { visibilisation of the } \\
\text { DMEK (Oct 2014), } \\
\text { anterior chamber flar } \\
\text { 2014), perforatin }\end{array}$ \\
\hline
\end{tabular}

Table 1. Characteristics of the studied cases. 